\title{
Animal Genetics
}

National Cancer Institute

\section{Source}

National Cancer Institute. Animal Genetics. NCI Thesaurus. Code C18633.

The study of genetic traits in animals. 